\title{
CLUSTER CONTINUOUS TIME RANDOM WALKS
}

\author{
A. JURLEWICZ, M. M. MEERSCHAERT, AND H.-P. SCHEFFLER
}

\begin{abstract}
In a continuous time random walk (CTRW), a random waiting time precedes each random jump. The CTRW model is useful in physics, to model diffusing particles. Its scaling limit is a time-changed process, whose densities solve an anomalous diffusion equation. This paper develops limit theory and governing equations for cluster CTRW, in which a random number of jumps cluster together into a single jump. The clustering introduces a dependence between the waiting times and jumps that significantly affects the asymptotic limit. Vector jumps are considered, along with oracle CTRW, where the process anticipates the next jump.
\end{abstract}

\section{INTRODUCTION}

The continuous time random walk (CTRW) is a useful model from statistical physics, in which each random particle jump is preceded by a random waiting time. Mathematically, the CTRW is a random walk subordinated to a renewal process. For $d$-dimensional vector jumps, the waiting times and jumps together form an i.i.d. sequence of $d+1$ dimensional random vectors, allowing dependence between the waiting time and the subsequent jump. The dependence is important in physics, for example, to ensure that particle velocities do not exceed the speed of light [22]. Coupling can also arise from clustering, where a random number of waiting times are combined, and the resulting jump is the sum of the clustered jump variables. This paper develops limit theory and governing equations for clustered CTRW. Since the waiting time and the subsequent jump in the cluster CTRW are both random sums, with the same random number of summands, the cluster CTRW is coupled, even if the original CTRW before clustering had no dependence between waiting times and jumps. If the number of jumps in a cluster has a heavy tail distribution, then the effect of clustering on the limit distribution, and the governing equation, can be profound. Section 2 introduces the required concepts from vector limit theory with matrix normalization, since the problem is inherently multidimensional, with dependence between coordinates. Section 3 applies vector limit theory to derive the long-time scaling limit process for cluster CTRW and cluster oracle CTRW (OCTRW) models. Governing equations

\footnotetext{
${ }^{1} 2010$ Mathematics Subject Classification. Primary: 60G50, 60F17, 60G18 and 60G22 ; Secondary: $60 \mathrm{H} 30,82 \mathrm{C} 31$.

Date: May 9, 2011.

Key words and phrases. continuous time random walk, anomalous diffusion equation, central limit theory, operator stable law.

MMM was partially supported by NSF grants DMS-0125486, DMS-0803360, EAR-0823965 and NIH grant R01-EB012079-01.
} 
are developed in Section 4, using the theory of pseudo-differential operators. Finally, Section 5 contains some examples, to illustrate the results of this paper.

\section{Continuous time Random Walks}

Let $J_{1}, J_{2}, \ldots$ be nonnegative independent and identically distributed (i.i.d) random variables that model the waiting times between jumps of a particle. We set $T(0)=0$ and $T(n)=\sum_{j=1}^{n} J_{j}$, the time of the $n$th jump. The particle jumps are given by i.i.d. random vectors $Y_{1}, Y_{2}, \ldots$ on $\mathbb{R}^{d}$. Let $S_{0}=0$ and $S(n)=\sum_{i=1}^{n} Y_{i}$, the position of the particle after the $n$th jump. For $t \geq 0$ let

$$
N_{t}=\max \{n \geq 0: T(n) \leq t\},
$$

the number of jumps up to time $t$ and define

$$
S\left(N_{t}\right)=\sum_{i=1}^{N_{t}} Y_{i}
$$

the position of a particle at time $t$. The stochastic process $\left\{S\left(N_{t}\right)\right\}_{t \geq 0}$ is called a continuous time random walk (CTRW). In some applications, it is also useful to consider the oracle CTRW

$$
S\left(N_{t}+1\right)=\sum_{i=1}^{N_{t}+1} Y_{i}
$$

which includes one additional jump.

Assume that $J_{1}$ belongs to the strict domain of attraction of some stable law with index $0<\beta<1$. This means that there exist $b_{n}>0$ such that

$$
b_{n}\left(J_{1}+\cdots+J_{n}\right) \Rightarrow D
$$

where $D>0$ almost surely. Here $\Rightarrow$ denotes convergence in distribution. The distribution $\lambda$ of $D$ is strictly stable with index $\beta$, meaning that $\lambda^{t}=t^{1 / \beta} \lambda$ for all $t>0$, where $\lambda^{t}$ is the $t$-th convolution power of the infinitely divisible law $\lambda$ and $(a \lambda)\{d x\}=\lambda\left\{a^{-1} d x\right\}$ is the probability distribution of $a D$ for $a>0$.

For $t \geq 0$ let $T(t)=\sum_{j=1}^{[t]} J_{j}$ and let $b(t)=b_{[t]}$, where $[t]$ denotes the integer part of $t$. Then $b(t)=t^{-1 / \beta} L(t)$ for some slowly varying function $L(t)$ and it follows from Example 11.2.18 of [16] that

$$
\{b(c) T(c t)\}_{t \geq 0} \stackrel{f . d .}{\Longrightarrow}\{D(t)\}_{t \geq 0} \quad \text { as } c \rightarrow \infty,
$$

where $\{D(t)\}$ is a $\beta$-stable subordinator such that $D(1)=D$. Here $\stackrel{f . d .}{\Longrightarrow}$ denotes convergence in distribution of all finite dimensional marginal distributions. 
Assume that $\left(Y_{i}\right)$ are i.i.d. $\mathbb{R}^{d}$-valued random variables independent of $\left(J_{i}\right)$ and that for some sequence of $d \times d$ matrices $B(n)$ with real entries we have

$$
B(n) \sum_{i=1}^{n} Y_{i} \Rightarrow A \quad \text { as } n \rightarrow \infty .
$$

To avoid trivial cases, we also assume that the distribution of $A$ is full, i.e., not supported on any lower dimensional hyperplane. Then we say that the distribution of $Y_{i}$ belongs to the (strict) generalized domain of attraction of $A$. Here strict refers to the fact that we did assume that there are no shifts needed in (2.6). By Theorem 8.1 .5 of [16] we can choose the sequence $B(n)$ to vary regularly with some index $-F$, meaning that $B([\lambda n]) B(n)^{-1} \rightarrow \lambda^{-F}$ as $n \rightarrow \infty$ for all $\lambda>0$, where $\lambda^{-F}=$ $\exp (-F \log \lambda)$ and $\exp (C)=I+C+C^{2} / 2 !+\cdots$ is the usual matrix exponential. In this case we will also write $B \in \mathrm{RV}(-F)$. The limit $A$ is (strictly) operator stable with exponent $F$, meaning that if $\left(A_{i}\right)$ are i.i.d. with $A$, then $n^{F} A$ and $A_{1}+\cdots+A_{n}$ are identically distributed for all positive integers $n$. Moreover, if we define the stochastic process $\{S(t)\}_{t \geq 0}$ by $S(t)=\sum_{i=1}^{[t]} Y_{i}$ it follows from Example 11.2.18 in [16] that

$$
\{B(c) S(c t)\}_{t \geq 0} \stackrel{f . d .}{\Longrightarrow}\{A(t)\}_{t \geq 0} \quad \text { as } c \rightarrow \infty,
$$

where $\{A(t)\}$ has stationary independent increments with $A(0)=0$ almost surely and $A(1)=A$. If $\mathbf{S}$ is a complete separable metric space, let $\mathbf{D}([0, \infty), \mathbf{S})$ denote the space of all right-continuous $\mathbf{S}$-valued functions on $[0, \infty)$ with limits from the left. Note that we can assume without loss of generality that sample paths of the processes $\{T(t)\}$ and $\{D(t)\}$ belong to $\mathbf{D}([0, \infty),[0, \infty))$, and that sample paths of $\{S(t)\}$ and $\{A(t)\}$ belong to $\mathbf{D}\left([0, \infty), \mathbb{R}^{d}\right)$. We can strengthen (2.7) using [17, Theorem 4.1] to get that

$$
\{B(c) S(c t)\}_{t \geq 0} \Rightarrow\{A(t)\}_{t \geq 0} \quad \text { as } c \rightarrow \infty,
$$

in the $J_{1}$ topology on $\mathbf{D}\left([0, \infty), \mathbb{R}^{d}\right)$.

Define the hitting time process of the stable subordinator $\{D(t)\}_{t \geq 0}$ by

$$
E(t)=\inf \{x \geq 0: D(x)>t\} .
$$

For $x, t \geq 0$ we have

$$
\{E(t) \leq x\}=\{D(x) \geq t\} .
$$

Since $b$ varies regularly with index $-1 / \beta$, there exists another regularly varying function $\tilde{b}$ with index $\beta$ such that $1 / b(\tilde{b}(c)) \sim c$ as $c \rightarrow \infty$. Here we use the notation $f \sim g$ for positive functions $f, g$ if and only if $f(c) / g(c) \rightarrow 1$ as $c \rightarrow \infty$. Define $\tilde{B}(c)=B(\tilde{b}(c))$. Recall from $(2.2)$ that $S\left(N_{t}\right)$ is the CTRW random variable that gives the location of a particle at time $t>0$. Then [17, Theorem 4.2] shows that

$$
\left\{\tilde{B}(c) S\left(N_{c t}\right)\right\}_{t \geq 0} \Rightarrow\{A(E(t))\}_{t \geq 0} \quad \text { in } \mathbf{D}\left([0, \infty), \mathbb{R}^{d}\right) \text { as } c \rightarrow \infty
$$


in the $M_{1}$-topology. Let $g_{\beta}(t)$ denote the smooth density of $D$. Using $(2.10)$ it is not hard to show that $E(t)$ has a density

$$
f_{t}(x)=\frac{t}{\beta} x^{-1-1 / \beta} g_{\beta}\left(t x^{-1 / \beta}\right)
$$

for any $t>0$, see [17, Corollary 3.1]. Since $A(t)$ is operator stable, it also has a smooth density $p(x, t)$ for all $t>0$, see [9, Theorem 4.10.2]. Then a simple conditioning argument shows that the CTRW limit process $A(E(t))$ in $(2.11)$ has a density

$$
h(x, t)=\frac{t}{\beta} \int_{0}^{\infty} p(x, u) g_{\beta}\left(t u^{-1 / \beta}\right) u^{-1 / \beta-1} d u
$$

for all $t>0$.

The linear operators $T_{t} f(x)=\int f(x-y) p(y, t) d y$ form a strongly continuous convolution semigroup with generator $L=\lim _{t \downarrow 0} t^{-1}\left(T_{t}-T_{0}\right)$, and $q(x, t)=T_{t} f(x)$ solves the abstract Cauchy problem $\partial_{t} q(x, t)=L q(x, t) ; q(x, 0)=f(x)$ for any initial condition $f(x)$ in the domain of the generator $L$, see for example $[6,7,20]$. Theorem 5.1 in [17] shows that the CTRW limit density $h(x, t)$ in $(2.13)$ solves the fractional Cauchy problem

$$
\partial_{t}^{\beta} h(x, t)=L h(x, t)+\delta(x) \frac{t^{-\beta}}{\Gamma(1-\beta)} .
$$

Here $\partial_{t}^{\beta} h(t, x)$ is the Riemann-Liouville fractional derivative, which can be defined as the inverse Laplace transform of $s^{\beta} \tilde{h}(x, s)$ when $\tilde{h}(x, s)=\int_{0}^{\infty} e^{-s t} h(x, t) d t$ is the usual Laplace transform in the time variable. Hence CTRW scaling limits are related to time-fractional differential equations, and the power-law index of the waiting times equals the order of the fractional time derivative.

\section{Cluster CTRW}

Now we define a new CTRW by clustering the space-time random vectors $X_{i}=$ $\left(Y_{i}, J_{i}\right)$. Assume that the i.i.d. cluster size random variables $M_{i}$, independent of $\left(X_{i}\right)$, take values on the nonnegative integers. Suppose that $\left(M_{i}\right)$ belongs to the strict domain of attraction of some stable law $Z$ with index $0<\gamma<1$ (see definition (2.4)), and note that in this case we have $\mathbb{E}\left(M_{i}\right)=\infty$. Let $C(n)=\sum_{i=1}^{n} M_{i}$ with $C(0)=0$ and define

$$
V_{n}=\sum_{i=C(n-1)+1}^{C(n)} X_{i}
$$

so that $V_{n}$ is a sequence of IID space-time jumps formed by summing $M_{n}$ consecutive space-time jumps from the original CTRW. Write $V_{i}=\left(Y_{i}^{M}, J_{i}^{M}\right)$ so that $J_{1}^{M}, J_{2}^{M}, \ldots$ are nonnegative i.i.d. random variables that model the waiting times between jumps of a particle in the cluster CTRW model. Set $T^{M}(0)=0$ and $T^{M}(n)=\sum_{j=1}^{n} J_{j}^{M}$, the time of the $n$th jump. The particle jumps are given by the i.i.d. random vectors 
$Y_{1}^{M}, Y_{2}^{M}, \ldots$ on $\mathbb{R}^{d}$. Let $S^{M}(0)=0$ and $S^{M}(n)=\sum_{i=1}^{n} Y_{i}^{M}$, the position of the particle after the $n$th jump in the cluster CTRW. For $t \geq 0$ let

$$
N_{t}^{M}=\max \left\{n \geq 0: T^{M}(n) \leq t\right\},
$$

the number of jumps up to time $t$ and define

$$
S^{M}\left(N_{t}^{M}\right)=\sum_{i=1}^{N_{t}^{M}} Y_{i}^{M}
$$

the position of a particle at time $t$. The stochastic process $\left\{S^{M}\left(N_{t}^{M}\right)\right\}_{t \geq 0}$ is called a cluster CTRW. The waiting time $J_{i}^{M}$ and the subsequent jump $Y_{i}^{M}$ are dependent random variables, since they both depend on the cluster size $M_{i}$, so that the cluster CTRW is a special case of the coupled CTRW considered in [3, 19]. In certain applications it is also useful to consider the cluster oracle CTRW $S^{M}\left(N^{M}(t)+1\right)$, in which one additional jump is included. In finance, the OCTRW represents the price at the next available trading time [12]. In geophysics, the OCTRW can represent the accumulated energy released during the next earthquake, or volcanic eruption, or the magnitude of the next flood event. The cluster CTRW and cluster OCTRW were introduced to model dielectric relaxation phenomena [25]. They both provide the model covering whole range of the observed (typical, as well as less typical) twopower-law relaxation behavior $[11,25,26]$. The clustering procedure is considered here as a stochastic generalization of the renormalization-group transformation idea, appearing frequently in physics and material science [5, 28], and applied to random walks in $[23,24]$. The goal of this paper is to study scaling limits and governing equations for the cluster CTRW and cluster OCTRW.

Since $M_{i}$ belongs to the strict domain of attraction of some stable law $Z$ with index $0<\gamma<1$, there exist $q_{n}>0$ such that

$$
q_{n}\left(M_{1}+\cdots+M_{n}\right) \Rightarrow Z
$$

where $Z>0$ almost surely. Note that we can choose $q_{n}$ in (3.3) so that $\mathbb{E}\left[e^{-s Z}\right]=e^{-s^{\gamma}}$. For $t \geq 0$ let $C(t)=\sum_{j=1}^{[t]} M_{j}$. Then $q(t)=q_{[t]}$ varies regularly with index $-1 / \gamma$, and it follows from Example 11.2.18 of [16] that

$$
\{q(c) C(c t)\}_{t \geq 0} \stackrel{\text { f.d. }}{\Longrightarrow}\{Z(t)\}_{t \geq 0} \quad \text { as } c \rightarrow \infty
$$

where $\{Z(t)\}$ is a $\gamma$-stable subordinator such that $Z(1)=Z$. Define

$$
K(t)=\max \{n \geq 0: C(n) \leq t\}
$$

Recall that $\left\{N_{t} \geq m\right\}=\{T(m) \leq t\}$. Then

$$
K\left(N_{t}\right)=\max \left\{n \geq 0: C(n) \leq N_{t}\right\}=\max \{n \geq 0: T(C(n)) \leq t\}=N_{t}^{M} .
$$

so that we can also write the clustered CTRW $S^{M}\left(N_{t}^{M}\right)=S\left(C\left(K\left(N_{t}\right)\right)\right)$ and the clustered OCTRW $S^{M}\left(N_{t}^{M}+1\right)=S\left(C\left(K\left(N_{t}\right)+1\right)\right)$. Note that $C(K(t))$ is itself a tightly coupled CTRW, with jumps equal to the waiting times. This process has been 
extensively studied in connection with the generalized arc sine distributions, see for example $[4,6]$. Define the inverse process

$$
R(t)=\inf \{x>0: Z(x)>t\}
$$

to the stable subordinator $Z(t)$. Let $U(t)=Z(R(t)-)$ and $O(t)=Z(R(t))$, the socalled undershoot and overshoot processes for the stable subordinator $\{Z(t)\}$. Recall from Section 2 that $\tilde{B}(c)=B(\tilde{b}(c))$.

Theorem 3.1. Assume that $\left(J_{i}\right)$ are i.i.d. satisfying $(2.4),\left(Y_{i}\right)$ are i.i.d. satisfying (2.6) and $\left(M_{i}\right)$ are i.i.d. satisfying (3.3). We also assume that $\left(J_{i}\right),\left(Y_{i}\right)$ and $\left(M_{i}\right)$ are independent. Then, using the notations above, the cluster CTRW scaling limit is given by

$$
\left\{\tilde{B}(c) S^{M}\left(N_{c t}^{M}\right)\right\}_{t \geq 0} \stackrel{f . d .}{\Longrightarrow}\{A(U(E(t)))\}_{t \geq 0}
$$

as $c \rightarrow \infty$. Also, the cluster OCTRW scaling limit is given by

$$
\left\{\tilde{B}(c) S^{M}\left(N_{c t}^{M}+1\right)\right\}_{t \geq 0} \stackrel{f . d_{.}}{\Longrightarrow}\{A(O(E(t)))\}_{t \geq 0}
$$

as $c \rightarrow \infty$.

Proof. Take $\tilde{q}(c)$ regularly varying with index $\gamma$ such that $1 / q(\tilde{q}(c)) \sim c$ as $c \rightarrow \infty$. In view of (3.3), it follows from [17, Corollary 3.4] that $\left\{\tilde{q}(c)^{-1} K(c t)\right\} \Rightarrow\{R(t)\}$ in the $J_{1}$ topology on $D([0, \infty),[0, \infty))$. Then it follows from [13, Theorem 3.1] with $q(\tilde{q}(c)) \sim 1 / c$ that

$$
\left\{c^{-1} C(K(c t)+1)\right\} \Rightarrow\{O(t)\} \quad \text { and } \quad\left\{c^{-1} C(K(c t))\right\} \Rightarrow\{U(t)\}
$$

as $c \rightarrow \infty$ in the $J_{1}$ topology on $\mathbf{D}([0, \infty), \mathbb{R})$. Note that the sample paths of the process $\{U(t)\}$ are modified at jumps, to make it right continuous. From Bertoin $[4$, III, Theorem 4] or [13, Example 5.2] we get $P(O(t)>t)=P(U(t)<t)=1$ for any $t>0$. Then $P(U(t)<t<O(t))=1$ for any $t>0$, since the intersection of two events of probability one also has probability one. If $U(t)<t<O(t)$ then $Z(x-)<t<Z(x)$ where $x=R(t)$, which implies that $x=R\left(t^{\prime}\right)$ for all $Z(x-)<t^{\prime}<Z(x)$, so that both $U\left(t^{\prime}\right)$ and $O\left(t^{\prime}\right)$ remain constant in a neighborhood of $t$. It follows that every $t>0$ is almost surely a continuity point of $U(t)$ and $O(t)$. Then it follows from [27, Theorem 11.6.6] that

$$
\left\{c^{-1} C(K(c t)+1)\right\} \stackrel{f . d .}{\Longrightarrow}\{O(t)\} \quad \text { and } \quad\left\{c^{-1} C(K(c t))\right\} \stackrel{f . d .}{\Longrightarrow}\{U(t)\} .
$$

Note that since $\left(M_{i}\right)$ and $\left(Y_{i}\right)$ are independent, (2.7) along with (3.7) implies

$$
\left\{\left(B(c) S(c t), c^{-1} C(K(c t)+1)\right)\right\} \stackrel{f . d .}{\Longrightarrow}\{(A(t), O(t))\}
$$

along with

$$
\left\{\left(B(c) S(c t), c^{-1} C(K(c t))\right)\right\} \stackrel{f . d .}{\Longrightarrow}\{(A(t), U(t))\}
$$


Then, arguing as in the proof of Theorem 4.1 in [2], using a transfer theorem, we get

$$
\left\{\left(B(c) S\left(c c^{-1} C(K(c t)+1)\right)\right)\right\} \stackrel{f . d .}{\Longrightarrow}\{A(O(t))\}
$$

as well as

$$
\{(B(c) S(C(K(c t))))\} \stackrel{f . d .}{\Longrightarrow}\{A(U(t))\} .
$$

By [17, Theorem 3.2] we have $\left\{\tilde{b}(c)^{-1} N_{c t}\right\} \stackrel{f . d .}{\Longrightarrow}\{E(t)\}$. Then another application of [2, Proposition 4.1] yields (3.5) and (3.6).

Remark 3.2. Recall that a stochastic process $\{X(t)\}$ is operator self-similar with exponent $P$ if

$$
\{X(c t)\} \stackrel{f . d .}{=}\left\{c^{P} X(t)\right\} \quad \text { for all } c>0,
$$

see for example the recent book of Embrechts and Maejima [15]. If $P=p I$, we also say that $\{X(t)\}$ is self-similar with index $p$. Hudson and Mason [8] showed that $\{A(t)\}$ is operator self-similar with exponent $F$, where $F$ is an exponent of the operator stable law $A$. Proposition 3.1 in [17] shows that $\{E(t)\}$ is self-similar with index $\beta$, and it follows from [13, Corollary 3.3] that both $\{U(t)\}$ and $\{O(t)\}$ are self-similar with index 1 . Then a simple conditioning argument yields

$$
\{A(U(E(c t)))\} \stackrel{f . d .}{=}\left\{A\left(U\left(c^{\beta} E(t)\right)\right)\right\} \stackrel{f . d .}{=}\left\{A\left(c^{\beta} U(E(t))\right)\right\} \stackrel{f . d .}{=}\left\{c^{\beta F} A(U(E(t)))\right\}
$$

and similarly for $A(O(E(t)))$, so that both limits in Theorem 3.1 are operator selfsimilar with exponent $\beta F$. Recall that

$$
A(t) \stackrel{d}{=} t^{F} A
$$

since $A$ is strictly operator stable. From [17, Corollary 3.1$]$ we get

$$
E(t) \stackrel{d}{=}(D / t)^{-\beta}
$$

where $D$ is stable with index $0<\beta<1$. From [13, Example 5.2] we get that

$$
U(t) \stackrel{d}{=} t \mathcal{B} \quad \text { and } \quad O(t) \stackrel{d}{=} t / \mathcal{B}
$$

where $\mathcal{B}$ has a beta density with parameters $\gamma$ and $1-\gamma$ (i.e., generalized arc sine distribution). Then we also have

$$
\begin{aligned}
\tilde{B}(c) S^{M}\left(N_{c t}^{M}\right) & \Rightarrow t^{\beta F} \mathcal{B}^{F} D^{-\beta F} A \\
\tilde{B}(c) S^{M}\left(N_{c t}^{M}+1\right) & \Rightarrow t^{\beta F} \mathcal{B}^{-F} D^{-\beta F} A
\end{aligned}
$$

for each $t>0$. This extends results in $[10,11,25,26]$ to the case of vector jumps.

Recall that the operator stable random vector $A(t)$ has a smooth density $p(x, t)$, and that the inverse subordinator $E(t)$ has density $f_{t}(x)$ given by $(2.12)$ in terms of the density $g_{\beta}(t)$ of $D$. Example 5.2 in [13] shows that $U(t)$ has density

$$
c(x, t)=\frac{x^{\gamma-1}(t-x)^{-\gamma}}{\Gamma(\gamma) \Gamma(1-\gamma)}, \quad 0<x<t
$$


and $O(t)$ has density

$$
a(x, t)=\frac{x^{-1}}{\Gamma(\gamma) \Gamma(1-\gamma)}\left(\frac{t}{x-t}\right)^{\gamma}, \quad x>t .
$$

Corollary 3.3. Under the assumptions of Theorem 3.1, the cluster CTRW limit $A(U(E(t)))$ has density

$$
p_{U}(x, t)=\int_{0}^{\infty} \int_{0}^{\infty} p(x, u) c(u, s) f_{s}(t) d u d s
$$

and the cluster OCTRW limit $A(O(E(t)))$ has density

$$
p_{O}(x, t)=\int_{0}^{\infty} \int_{0}^{\infty} p(x, u) a(u, s) f_{s}(t) d u d s .
$$

Proof. This is a simple conditioning argument, since the three processes in the limits in each of (3.5) and (3.6) are independent.

Remark 3.4. As a simple extension of Theorem 3.1 we can consider waiting times with a finite mean $m=\mathbb{E} J_{1}<\infty$. Now (2.4) holds with $b_{n}=1 /(n m)$ by the law of large numbers, and $D(t)=E(t)=t$. Then the CTRW limit $A(t)$ has a smooth density $p(x, t)$ that solves the Cauchy problem $\partial_{t} p(x, t)=L p(x, t)$. The cluster CTRW limit is $A(U(t))$ and the cluster OCTRW limit is $A(O(t))$. The limit densities are given by Corollary 3.3 with $f_{s}(t)=\delta(t-s)$.

There is another useful way to view the cluster CTRW in terms of coupled random sums. Note that $S^{M}(n)=S(C(n))$ and $T^{M}(n)=T(C(n))$ are both random sums, and $T^{M}(n)$ has inverse $N_{t}^{M}=K\left(N_{t}\right)$. The space-time jumps $X_{i}=\left(Y_{i}, J_{i}\right)$ are i.i.d. random vectors in $\mathbb{R}^{d} \times[0, \infty)$ that belong to the strict generalized domain of attraction of some full operator stable random vector $W=(A, D)$ with distribution $\mu$, and

$$
\left(B(n) \sum_{i=1}^{n} Y_{i}, b_{n} \sum_{i=1}^{n} J_{i}\right) \Rightarrow(A, D)
$$

so that

$$
\{(B(c) S(c t), b(c) T(c t))\}_{t \geq 0} \stackrel{f . d .}{\Longrightarrow}\{(A(t), D(t))\}_{t \geq 0} \quad \text { as } c \rightarrow \infty
$$

in view of [16, Example 11.2.18]. Note that $W$ is strictly operator stable with exponent $H=\operatorname{diag}(F, 1 / \beta)$, and that $(B(c), b(c))$ varies regularly at infinity with index $-H$. Next we need an extension of some technical results on operator $\nu$-stable laws from [14]. Operator $\nu$-stable laws are limits of random sums of i.i.d. random vectors in the generalized domain of attraction of an operator stable law. Recall that $V_{i}$ are the cluster jumps in space-time, each of which is given by a random sum.

Theorem 3.5. Under the above conditions, $\left(V_{i}\right)$ belongs to the strict generalized domain of attraction of some full operator stable random vector $W(Z)$ with exponent $(1 / \gamma) H$. 
Proof. Recall that $\left(X_{i}\right)$ are in the strict generalized domain of attraction of some operator stable law $W$ on $\mathbb{R}^{d} \times[0, \infty)$ with exponent $H$, and that for $S_{X}(t)=\sum_{i=1}^{\lfloor t\rfloor} X_{i}$ we have

$$
\left\{Q(c) S_{X}(c t)\right\} \stackrel{f . d .}{\Longrightarrow}\{W(t)\} \quad \text { as } c \rightarrow \infty
$$

where $\{W(t)\}$ is a Lévy process generated by $W(1)=W$, and $Q(c)=(B(c), b(c))$ is regularly varying with index $-H$. Moreover, for some $q \in \operatorname{RV}(-1 / \gamma)$ we have $(3.4)$ where $\{Z(t)\}$ is a $\gamma$-stable subordinator with $Z(1)=Z$. Note that the random sum

$$
\sum_{j=1}^{n} V_{j}=\sum_{i=1}^{C(n)} X_{i}=S_{X}(C(n)) .
$$

Following the same line of proof as Theorem 3.1, using the transfer theorem from [2], we get using (3.15) and (3.4) that

$$
\left\{Q\left(q(c)^{-1}\right) S_{X}\left(q(c)^{-1} q(c) C(c t)\right)\right\} \stackrel{f . d .}{\Longrightarrow}\{W(Z(t))\}
$$

as $c \rightarrow \infty$. Especially, for $t=1$ and $c=n$ we get

$$
Q\left(q(n)^{-1}\right) \sum_{j=1}^{n} V_{j} \Longrightarrow W(Z)
$$

showing that $\left(V_{i}\right)$ belongs to the generalized domain of attraction of $W(Z)$. Using the self-similarity

$$
W(t) \stackrel{d}{=} t^{H} W \quad \text { and } \quad Z(t) \stackrel{d}{=} t^{1 / \gamma} Z,
$$

we get using independence again that

$$
W(Z(t)) \stackrel{d}{=} W\left(t^{1 / \gamma} Z\right) \stackrel{d}{=} t^{(1 / \gamma) H} W(Z)
$$

showing that $W(Z)$ is operator stable with exponent $(1 / \gamma) H$.

The following corollary interprets Theorem 3.5 in terms of regular variation of the underlying probability measures. Let $V=V_{1}$. We say that a probability measure $\mu$ varies regularly at infinity with index $H$ if we have

$$
n B(n) \mu(d x) \rightarrow \phi(d x) \quad \text { as } n \rightarrow \infty
$$

for some sequence of matrices $B \in \mathrm{RV}(-H)$, and some sigma-finite Borel measure $\phi$ that is not concentrated on any lower dimensional subspace. Here the convergence means that $n \mu\left\{B(n)^{-1} x: x \in U\right\} \rightarrow \phi(U)$ as $n \rightarrow \infty$ for Borel sets $U$ that are bounded away from the origin, and whose boundary has $\phi$-measure zero. In that case, we write $\mu \in \operatorname{RVM}_{\infty}(H)$, see Section 6.1 in [16].

Corollary 3.6. Theorem 3.5 implies that if $P_{X} \in \operatorname{RVM}_{\infty}(H)$, then $P_{V} \in$ $\operatorname{RVM}_{\infty}((1 / \gamma) H)$ 
Proof. By Theorem 8.2.10 of [16] we have $X \in \operatorname{GDOA}(W)$ for some full operator stable $W$ with exponent $H$ and no normal component if and only if $P_{X} \in \operatorname{RVM}_{\infty}(H)$. Now the result follows from Theorem 3.5.

Remark 3.7. Let $A^{M}(t)=A(Z(t))$ and $D^{M}(t)=D(Z(t))$ and note that (3.16) yields

$$
\left\{\left(B\left(q(c)^{-1}\right) S_{X}(C(c t)), b\left(q(c)^{-1}\right) T(C(c t))\right)\right\} \stackrel{f . d .}{\Longrightarrow}\left\{\left(A^{M}(t), D^{M}(t)\right)\right\}
$$

as $c \rightarrow \infty$. If we define

$$
E^{M}(t)=\inf \left\{x>0: D^{M}(x)>t\right\}
$$

then it is easy to check that $E^{M}(t)=R(E(t))$, so that the cluster CTRW limit in Theorem 3.1 is

$$
A(U(E(t)))=A(Z(R(E(t)-)))=A^{M}\left(E^{M}(t)-\right)
$$

and the cluster OCTRW limit in Theorem 3.1 is

$$
A(O(E(t)))=A(Z(R(E(t))))=A^{M}\left(E^{M}(t)\right) .
$$

The convergence (3.17) does not lead directly to the results of Theorem 3.1 because $A^{M}(t), D^{M}(t)$ are not independent. However, it will be useful in the next section.

\section{Governing EQUATions}

This section develops governing equations for the cluster CTRW and OCTRW limits. Since the underlying process is a coupled CTRW, we follow the development in $[3,13]$. For suitable functions $f$ on $\mathbb{R}^{d} \times \mathbb{R}_{+}$we define the Fourier-Laplace transform

$$
\bar{f}(k, s)=\int_{\mathbb{R}^{d}} \int_{0}^{\infty} e^{i\langle x, k\rangle} e^{-s t} f(x, t) d t d x
$$

where $(k, s) \in \mathbb{R}^{d} \times(0, \infty)$. Similarly, if $\mu$ is a bounded Borel measure on $\mathbb{R}^{d} \times \mathbb{R}_{+}$,

$$
\bar{\mu}(k, s)=\int_{\mathbb{R}^{d}} \int_{0}^{\infty} e^{i\langle x, k\rangle} e^{-s t} \mu(d x, d t)
$$

is the Fourier-Laplace transform of $\mu$.

Any infinitely divisible distribution is characterized by the Lévy-Khinchin formula. This concept carries over to the FLT setting [3, Lemma 2.1] so that, if $(A, D)$ is an infinitely divisible random variable on $\mathbb{R}^{d} \times \mathbb{R}_{+}$with distribution $\mu$, we have

$$
\bar{\mu}(k, s)=\mathbb{E}\left[e^{-s D+i\langle k, A\rangle}\right]=e^{-\psi(k, s)}
$$

for all $(k, s) \in \mathbb{R} \times \mathbb{R}_{+}$. We call $\psi(k, s)$ the Fourier-Laplace symbol of $(A, D)$. Moreover, there exist uniquely determined $(a, b) \in \mathbb{R}^{d} \times \mathbb{R}_{+}$, a positive semi-definite matrix $P$ on $\mathbb{R}^{d}$ and a measure $\phi$ on $\mathbb{R}^{d} \times \mathbb{R}_{+} \backslash\{(0,0)\}$ such that

$$
\begin{aligned}
\psi(k, s)= & i\langle a, k\rangle+b s+\langle k, P k\rangle \\
& +\int_{\mathbb{R}^{d} \times \mathbb{R}_{+} \backslash\{(0,0)\}}\left(1-e^{i\langle k, x\rangle} e^{-s t}+\frac{i\langle k, x\rangle}{1+\|x\|^{2}}\right) \phi(d x, d t) .
\end{aligned}
$$


The Lévy measure $\phi$ is finite outside every neighborhood of the origin and

$$
\int_{0<\|x\|^{2}+t \leq 1}\left(\|x\|^{2}+t\right) \phi(d x, d t)<\infty .
$$

We denote by $\phi_{A}(d x)=\phi\left(d x, \mathbb{R}_{+}\right)$the Lévy measure of $A$. By setting $s=0$ in the representation (4.2) we see that $\mathbb{E}\left[e^{i\langle k, A\rangle}\right]=e^{-\psi_{A}(k)}$ and we call

$$
\psi_{A}(k)=i\langle a, k\rangle+\langle k, P k\rangle+\int_{\mathbb{R}^{d} \backslash\{0\}}\left(1-e^{-i\langle k, x\rangle}+\frac{i\langle k, x\rangle}{1+\|x\|^{2}}\right) \phi_{A}(d x)
$$

the Fourier symbol of $A$. Similarly, we let $\phi_{D}(d t)=\phi(\mathbb{R}, d t)$ denote the Lévy measure $D$. By setting $k=0$ in the representation (4.2) we see that $\mathbb{E}\left[e^{-s D}\right]=e^{-\psi_{D}(s)}$ and we call

$$
\psi_{D}(s)=b s+\int_{0}^{\infty}\left(1-e^{-s v}\right) \phi_{D}(d v)
$$

the Laplace symbol of $D$. If $A, D$ are independent, then $\psi(k, s)=\psi_{A}(k)+\psi_{D}(s)$.

Given any $\lambda>0$ let $L_{\lambda}^{1}\left(\mathbb{R}^{d} \times \mathbb{R}_{+}\right)$denote the collection of real-valued measurable functions on $\mathbb{R}^{d} \times \mathbb{R}_{+}$for which the integral and hence the norm

$$
\|f\|_{\lambda}=\int_{0}^{\infty} \int_{\mathbb{R}^{d}} e^{-\lambda t}|f(x, t)| d x d t
$$

exists. With this norm, $L_{\lambda}^{1}\left(\mathbb{R}^{d} \times \mathbb{R}_{+}\right)$is a Banach space that contains $L^{1}\left(\mathbb{R}^{d} \times \mathbb{R}_{+}\right)$. The symbol $\psi(k, s)$ defines a pseudo-differential operator $\psi\left(i D_{x}, \partial_{t}\right)$ on this space, and the negative generator of the corresponding Feller semigroup, see [19] for more details. Theorem 3.2 in [1] shows that the domain of this operator contains any $f \in L_{\lambda}^{1}\left(\mathbb{R}^{d} \times \mathbb{R}_{+}\right)$whose weak first and second order spatial derivatives as well as weak first order time derivatives are in $L_{\lambda}^{1}\left(\mathbb{R}^{d} \times \mathbb{R}_{+}\right)$, and that in this case we have

$$
\begin{aligned}
& \psi\left(i D_{x}, \partial_{t}\right) f(x, t)=-a \nabla_{x} f(x, t)+b \partial_{t} f(x, t)-\left\langle\nabla_{x}, P \nabla_{x} f(x, t)\right\rangle \\
& -\int_{\mathbb{R} \times \mathbb{R}_{+} \backslash\{(0,0)\}}\left(H(t-u) f(x-y, t-u)-f(x, t)+\frac{\left\langle y, \nabla_{x} f(x, t)\right\rangle}{1+\|y\|^{2}}\right) \phi(d y, d u)
\end{aligned}
$$

where $\nabla_{x}=\left(\partial_{x_{1}}, \ldots, \partial_{x_{d}}\right)^{\top}$ and $H(t)=I(t \geq 0)$ is the Heaviside step function. It follows that $\psi(k, s) \bar{f}(k, s)$ is the Laplace-Fourier transform of $\psi\left(i D_{x}, \partial_{t}\right) f(x, t)$ for any $f \in L_{\lambda}^{1}\left(\mathbb{R}^{d} \times \mathbb{R}_{+}\right)$.

As in $[2,3,13,19]$ among other works, we say that a function $f(x, t)$ is a mild solution of a pseudo-differential equation

$$
\psi\left(i D_{x}, \partial_{t}\right) f(x, t)=g(x, t)
$$

for some distribution $g(x, t)$ if and only if

$$
\psi(k, s) \bar{f}(k, s)=\bar{g}(k, s)
$$

for all $k \in \mathbb{R}^{d}$ and $s>0$. That is, the Fourier-Laplace transform of $f$ in (4.5) solves the algebraic equation (4.6). 
Remark 4.1. It is a hard and open analytical problem to derive a general theory of strong (or weak) solutions of equations of the form (4.5), which goes far beyond the scope of this paper.

Recall from Theorem 3.5 that $W(Z)=\left(A^{M}, D^{M}\right)$ is an operator stable Lévy process with exponent $(1 / \gamma) H$. Next we compute its Fourier-Laplace symbol.

Lemma 4.2. If $\psi(k, s)$ is the Fourier-Laplace symbol of $(A, D)$ then the FourierLaplace symbol of $W(Z)=\left(A^{M}, D^{M}\right)$ is given by $\psi^{M}(k, s)=\psi(k, s)^{\gamma}$.

Proof. Note that we choose $q_{n}$ in (3.3) so that $\mathbb{E}\left[e^{-s Z}\right]=e^{-s^{\gamma}}$. Recall that $W(Z)=$ $(A(Z), D(Z))$ with $Z=Z(1)$ and write

$$
\mathbb{E}\left[e^{i\left\langle k, A^{M}\right\rangle-s D^{M}}\right]=\mathbb{E}\left[\mathbb{E}\left[e^{i\langle k, A(Z)\rangle-s D(Z)} \mid Z\right]\right]=\mathbb{E}\left[e^{-\psi(k, s) Z}\right]=e^{-\psi(k, s)^{\gamma}}
$$

using the independence of $\{(A(t), D(t))\}$ and $\{Z(t)\}$.

Recall that the cluster OCTRW limit $A^{M}\left(E^{M}(t)\right)$ has a density $p_{O}(x, t)$ given by (3.12), and the cluster CTRW limit $A^{M}\left(E^{M}(t)-\right)$ has a density $p_{U}(x, t)$ given by (3.11). Also recall that $\psi_{A}(k)$ is the Fourier symbol of the operator stable limit for the (non-clustered) jump variables. The next result gives the governing equations for the limit densities $p_{O}(x, t)$ and $p_{U}(x, t)$.

Theorem 4.3. Under the assumptions of Theorem 3.1, the cluster CTRW limit density (3.11) is a mild solution of

$$
\left(\psi_{A}\left(i D_{x}\right)+\partial_{t}^{\beta}\right)^{\gamma} p_{U}(x, t)=\delta(x) \frac{t^{-\beta \gamma}}{\Gamma(1-\beta \gamma)},
$$

and the cluster OCTRW limit density (3.12) is a mild solution of

$$
\left(\psi_{A}\left(i D_{x}\right)+\partial_{t}^{\beta}\right)^{\gamma} p_{O}(x, t)=\frac{1}{\Gamma(1-\gamma)} \int_{0}^{\infty} \nu^{u}(d x) \mathbb{P}(D(u)>t) \gamma u^{-\gamma-1} d u
$$

where $\nu^{u}$ is the probability distribution of the limit $A(u)$ in (2.6).

Proof. We follow the development in [13]. Theorem 3.1 along with Remark 3.7 shows that $A^{M}\left(E^{M}(t)\right)$ is the cluster OCTRW limit, and $A^{M}\left(E^{M}(t)-\right)$ is the cluster CTRW limit. Let $\psi_{A(Z)}(k)=\psi^{M}(k, 0)$ be the Fourier symbol of $A(Z)$ and let $\psi_{D(Z)}(s)=$ $\psi^{M}(0, s)$ be the Laplace symbol of $D(Z)$. Although [13, Theorem 4.1] is stated and proved in the special case of scalar jumps, the proof extends immediately to vector jumps. Then it follows that the cluster OCTRW limit has Fourier-Laplace transform

$$
\bar{p}_{O}(k, s)=\frac{1}{s} \frac{\psi^{M}(k, s)-\psi_{A(Z)}(k)}{\psi^{M}(k, s)},
$$

and the cluster CTRW limit has Fourier-Laplace transform

$$
\bar{p}_{U}(k, s)=\frac{1}{s} \frac{\psi_{D(Z)}(s)}{\psi^{M}(k, s)} .
$$


Since $A, D$ are independent, the operator stable random vector $(A, D)$ has FourierLaplace symbol $\psi(k, s)=\psi_{A}(k)+s^{\beta}$, and then it follows from Lemma 4.2 that $\psi^{M}(k, s)=\psi(k, s)^{\gamma}=\left(\psi_{A}(k)+s^{\beta}\right)^{\gamma}$. Then we can rearrange (4.10) to get

$$
\left(\psi_{A}(k)+s^{\beta}\right)^{\gamma} \bar{p}_{U}(k, s)=\frac{1}{s} \psi_{D(Z)}(s) .
$$

Next we want to invert the Fourier-Laplace transforms in (4.11). From [19, Eq. (3.12)] we get that $s^{-1} \psi_{D(Z)}(s)$ is the Laplace transform of $\phi_{D(Z)}(t, \infty)$. Since the subordinator $D(Z)$ has Laplace symbol $\psi_{D(Z)}(s)=\psi^{M}(0, s)=s^{\beta \gamma}$, a calculation similar to [16, Lemma 7.3.7] shows that

$$
\phi_{D(Z)}(t, \infty)=\frac{t^{-\beta \gamma}}{\Gamma(1-\beta \gamma)} .
$$

Then we can invert the Fourier-Laplace transforms in (4.11) to arrive at (4.7).

The argument for (4.8) is similar. From (4.9) we get

$$
\left(\psi_{A}(k)+s^{\beta}\right)^{\gamma} \bar{p}_{O}(k, s)=\frac{1}{s}\left(\psi^{M}(k, s)-\psi_{A(Z)}(k)\right) .
$$

The vector version of [13, Lemma 4.6] gives

$$
\int_{0}^{\infty} e^{-s t} \int_{\mathbb{R}} e^{i\langle k, x\rangle} \phi_{W(Z)}(d x,(t, \infty)) d t=\frac{1}{s}\left(\psi^{M}(k, s)-\psi_{A(Z)}(k)\right),
$$

and then we can invert (4.13) to arrive at

$$
\left(\psi_{A}\left(i D_{x}\right)+\partial_{t}^{\beta}\right)^{\gamma} p_{O}(x, t)=\phi_{W(Z)}(d x,(t, \infty)) .
$$

It follows from Sato [21, Theorem 30.1] that $W(Z)$ is infinitely divisible with Lévy measure

$$
\phi_{W(Z)}(d x, d t)=\int_{0}^{\infty} \mu^{u}(d x, d t) \phi_{Z}(d u)
$$

where $\mu^{u}$ is the distribution of $(A(u), D(u))$ and $\phi_{Z}$ is the Lévy measure of $Z$. Since $Z$ is a standard stable subordinator with Laplace symbol $s^{\gamma}$, it follows that $\phi_{Z}(t, \infty)=$ $t^{-\gamma} / \Gamma(1-\gamma)$. Since $A, D$ are independent, $\mu^{u}(d x, d t)=\nu^{u}(d x) \lambda^{u}(d t)$ where $\nu^{u}$ is the distribution of $A(u)$ and $\lambda^{u}$ is the distribution of $D(u)$. Then we have

$$
\phi_{W(Z)}(d x,(t, \infty))=\frac{1}{\Gamma(1-\gamma)} \int_{0}^{\infty} \nu^{u}(d x) \mathbb{P}(D(u)>t) \gamma u^{-\gamma-1} d u
$$

which can be substituted into (4.15) to obtain (4.8).

Remark 4.4. As in Remark 3.4, we can consider waiting times with a finite mean. Then $D(t)=t$ so that $\psi_{D}(s)=s$ and $\psi(k, s)=s+\psi_{A}(k)$. Lemma 4.2 still holds, (4.7) still applies with $\beta=1$, and (4.8) simplifies to

$$
\left(\psi_{A}\left(i D_{x}\right)+\partial_{t}\right)^{\gamma} p_{O}(x, t)=\frac{1}{\Gamma(1-\gamma)} \int_{t}^{\infty} \nu^{u}(d x) \gamma u^{-\gamma-1} d u
$$


since $\mathbb{P}(D(u)>t)=I(u>t)$ in this case.

\section{EXAMPLES}

Here we provide a few useful examples, to illustrate the results of this paper.

Example 5.1. Suppose that $\mathbb{E} Y_{1}=0$ and $\mathbb{E}\left\|Y_{1}\right\|^{2}<\infty$ so that (2.6) holds with $B(n)=2 n^{-1 / 2} M^{-1 / 2}$ where $M=\mathbb{E}\left[Y_{1} Y_{1}^{T}\right]$, and $A$ is multivariate normal with covariance matrix $2 I$. If $\mathbb{E} J_{1}<\infty$ then as in Remark 4.4 the cluster CTRW limit $A(U(t))$ has a density

$$
p_{U}(x, t)=\int_{0}^{\infty} p(x, u) c(u, t) d u
$$

where $p(x, u)=(4 \pi u)^{-d / 2} \exp \left(-\|x\|^{2} /(4 u)\right)$ is the density of $A(u)$, and $c(u, t)$ is given by (3.9). The governing equation is

$$
\left(\Delta+\partial_{t}\right)^{\gamma} p_{U}(x, t)=\delta(x) \frac{t^{-\gamma}}{\Gamma(1-\gamma)},
$$

since $\psi_{A}(k)=-\|k\|^{2}$ is the Fourier symbol of the Laplacian $\Delta=\partial_{x_{1}}^{2}+\cdots+\partial_{x_{d}}^{2}$. Note that $A(Z)$ is symmetric stable with index $2 \gamma$. If $d=1$ we get

$$
p_{U}(x, t)=\int_{0}^{t} \frac{1}{\sqrt{4 \pi u}} \exp \left(-\frac{x^{2}}{4 u}\right) \frac{u^{\gamma-1}}{\Gamma(\gamma)} \frac{(t-u)^{-\gamma}}{\Gamma(1-\gamma)} d u
$$

which is the same form as Example 5.2 in [3], since clustering induces the same dependence as the variance coupling in [3, Example 5.2]. That same model of variance coupling was applied in [18] to model price returns for bond futures. Hence this example also demonstrates that clustering is sufficient to explain the coupling observed in that data. The OCTRW limit in dimension $d=1$ has density

$$
p_{O}(x, t)=\int_{t}^{\infty} \frac{1}{\sqrt{4 \pi u}} \exp \left(-\frac{x^{2}}{4 u}\right) \frac{x^{-1}}{\Gamma(\gamma) \Gamma(1-\gamma)}\left(\frac{t}{x-t}\right)^{\gamma} d u
$$

and governing equation

$$
\left(\partial_{x}^{2}+\partial_{t}\right)^{\gamma} p_{O}(x, t)=\frac{1}{\Gamma(1-\gamma)} \int_{t}^{\infty} \frac{1}{\sqrt{4 \pi u}} \exp \left(-\frac{x^{2}}{4 u}\right) \gamma u^{-\gamma-1} d u
$$

which agrees with Example 5.3 in [13]. In the application from [18], the OCTRW represents the log-price of the bond futures at the next trading time, and the clustering variable counts the number of trades executed at that time.

Example 5.2. Suppose that (2.6) holds with $A$ symmetric stable, and $\mathbb{E}\left[e^{i\langle k, A(u)\rangle}\right]=$ $e^{-u \psi_{A}(k)}$ with $\psi_{A}(k)=-\|k\|^{\alpha}$ for some $0<\alpha<2$. If $\mathbb{E} J_{1}<\infty$ then, as in Remark 4.4, the cluster CTRW limit $A(U(t))$ has governing equation

$$
\left(\Delta^{\alpha / 2}+\partial_{t}\right)^{\gamma} p_{U}(x, t)=\delta(x) \frac{t^{-\gamma}}{\Gamma(1-\gamma)},
$$


where $\Delta^{\alpha / 2}$ is the fractional Laplacian with Fourier symbol $\psi_{A}(k)$. Here $A(Z)$ is symmetric stable with index $\alpha \gamma$. For infinite mean waiting times as in Theorem 3.1, the governing equation is

$$
\left(\Delta^{\alpha / 2}+\partial_{t}^{\beta}\right)^{\gamma} p_{U}(x, t)=\delta(x) \frac{t^{-\beta \gamma}}{\Gamma(1-\beta \gamma)},
$$

and $D(Z)$ is stable with index $\beta \gamma$. The OCTRW limit has governing equation (4.8) where $\nu^{u}(d x)$ is the symmetric stable distribution of $A(u)$. This extends Example 5.4 in [13] to vector jumps.

\section{REFERENCES}

[1] B. Baeumer, M.M. Meerschaert and J. Mortensen (2005) Space-time fractional derivative operators. Proc. Amer. Math. Soc. 133, No. 8, 2273-2282.

[2] P. Becker-Kern, M.M. Meerschaert and H.P. Scheffler (2004) Limit theorem for continuous time random walks with two time scales. J. Applied Probab. 41, No. 2, 455-466.

[3] P. Becker-Kern, M.M. Meerschaert and H.P. Scheffler (2004) Limit theorems for coupled continuous time random walks. The Annals of Probability 32, No. 1B, 730-756.

[4] J. Bertoin (1996) Lévy processes. Cambridge University Press.

[5] T.G. Dewey (1997) Fractals in Molecular Biophysics, Oxford University Press, Oxford.

[6] W. Feller (1971) An Introduction to Probability Theory and Its Applications. Vol. II, 2nd Ed., Wiley, New York.

[7] E. Hille and R.S. Phillips (1957) Functional Analysis and Semi-Groups. Amer. Math. Soc. Coll. Publ. 31, American Mathematical Society, Providence.

[8] Hudson, W. and J. D. Mason (1982) Operator-self-similar processes in a finite-dimensional space. Trans. Amer. Math. Soc. 273, 281-297.

[9] Z.J. Jurek and J.D. Mason (1993) Operator-Limit Distributions in Probability Theory. Wiley Interscience, New York.

[10] A. Jurlewicz (2005) Limit theorems for randomly coarse grained continuous-time random walks. Dissertationes Mathematicae 431, 1-45.

[11] A. Jurlewicz, K. Weron, and M. Teuerle (2008) Generalized Mittag-Leffler relaxation: Clustering-jump continuous-time random walk approach. Phys. Rev. E 78, 011103.

[12] A. Jurlewicz, A. Wyłomańska and P. Żebrowski (2009) Coupled continuous-time random walk approach to the Rachev-Rüschendorf model for financial data. Physica A 388, 407-418.

[13] A. Jurlewicz, P. Kern, M.M. Meerschaert and H.P. Scheffler (2010) Oracle continuous time random walks. Preprint available at www.stt.msu.edu/ mcubed/ACTRW.pdf

[14] T.J. Kozubowski, M.M. Meerschaert and H.P. Scheffler (2004) The operator $n u$-stable laws. Publ. Math. Debrecen 63, 569-585.

[15] P. Embrechts and M. Maejima (2002) Selfsimilar Processes, Princeton University Press, Princeton, New York.

[16] M.M. Meerschaert and H.P. Scheffler (2001) Limit Distributions for Sums of Independent Random Vectors: Heavy Tails in Theory and Practice. Wiley Interscience, New York.

[17] M.M. Meerschaert and H.P. Scheffler (2004) Limit theorems for continuous time random walks with infinite mean waiting times. J. Applied Probab. 41, No. 3, 623-638.

[18] M.M. Meerschaert and E. Scalas (2006) Coupled continuous time random walks in finance. Physica A: Statistical Mechanics and Its Applications 370, 114-118.

[19] M.M. Meerschaert and H.P. Scheffler (2008) Triangular array limits for continuous time random walks. Stoch. Proc. Appl. 118, 1606-1633. 
[20] A. Pazy (1983) Semigroups of Linear Operators and Applications to Partial Differential equations. Applied Mathematical Sciences 44, Springer-Verlag, New York.

[21] K.I. Sato (1999) Lévy Processes and Infinitely Divisible Distributions. Cambridge University Press.

[22] M. Shlesinger, J. Klafter and Y.M. Wong (1982) Random walks with infinite spatial and temporal moments. J. Statist. Phys. 27, 499-512.

[23] M.O. Vlad (1992) Hierarchical clustering-jump approach to analogs of renormalization-group transformations in fractal random processes. Phys. Rev. A 45, 36003614.

[24] M.O. Vlad and M.C. Mackey (1995) Stochastic renormalization-group approach to spacedependent supercritical branched chain processes. Phys. Rev. E 51, 31043119.

[25] K. Weron, A. Jurlewicz, and M. Magdziarz, Havriliak-Negami response in the framework of the continuous-time random walk. Acta Phys. Pol. B 36, 1855 (2005).

[26] K. Weron, A. Jurlewicz, M. Magdziarz, A. Weron, and J. Trzmiel (2010) Overshooting and Undershooting Subordination Scenario for Fractional Two-Power-Law Relaxation Responses. Phys. Rev. E 81, 041123.

[27] W. Whitt (2002) Stochastic Process Limits: An Introduction to Stochastic-Process Limits And their Application to Queues. Springer, New York.

[28] G. M. Zaslavsky (2002) Chaos, fractional kinetics, and anomalous transport. Physics Reports 371 (6), 461-580.

Agnieszka Jurlewicz, Hugo Steinhaus Center, Institute of Mathematics and Computer Science, Wroceaw University of Technology, Wroceaw, Poland

E-mail address: Agnieszka.Jurlewicz@pwr.wroc.pl

Mark M. Meerschaert, Department of Statistics and Probability, Michigan State University, EAST LANSING, MI 48824

E-mail address: mcubed@stt.msu.edu

URL: http://www.stt.msu.edu/ mcubed/

Hans-Peter Scheffler, Fachbereich Mathematik, Universität Siegen, 57068 Siegen, GERMAnY

E-mail address: scheffler@mathematik.uni-siegen.de

URL: http://www.stat.math. uni-siegen.de/ scheffler/ 